This document is the unedited Author's version of a Submitted Work that was subsequently accepted for publication in ACS Applied Materials \& Interfaces, (C American Chemical Society, after peer review. To access the final edited and published work see http://dx.doi.org/10.1021/acsami.8b01351

\title{
Composite Hydrogels using Bioinspired Approach with In Situ Fast Gelation and Self- Healing Ability as Future Injectable Biomaterial
}

\author{
Musammir Khan ${ }^{\dagger, \perp},{ }^{*}$, Janne T. Koivisto $^{\dagger,}$, , Terttu Hukka ${ }^{\ddagger}$, Mikko Hokka ${ }^{\S}$, and Minna \\ Kellomäki ${ }^{\dagger}, \|, *$ \\ ${ }^{\dagger}$ BioMediTech Institute and Faculty of Biomedical Sciences and Engineering, ${ }^{\dagger}$ Department of \\ Chemistry and Bioengineering, ${ }^{\S}$ Department of Material Science, Tampere University of \\ Technology, Korkeakoulunkatu 10, 33720 Tampere, Finland \\ "BioMediTech Institute and Faculty of Medicine and Life Sciences, University of Tampere, \\ Lääkärinkatu 1, 33520 Tampere, Finland \\ ${ }^{\perp}$ Institute of Chemical Sciences, University of Peshawar, Peshawar 25120, Pakistan
}




\begin{abstract}
Biopolymers are attractive candidates to fabricate biocompatible hydrogels, but the low water solubility of most of them at physiological $\mathrm{pH}$, has hindered their applications. To prepare a water-soluble derivative of chitosan (WSC) biopolymer, it was grafted with a small anionic amino acid, L-glutamic acid, using a single step 1-Ethyl-3-[3dimethylaminopropyl] carbodiimide coupling reaction. This resulted in a zwitterion-tethered structure onto the polymer backbone. The degree of substitution ranged between 13-16 \pm 1.25 $\%$, which was controlled by varying the feeding reagent ratios. The differential scanning calorimetry and X-ray diffraction based analysis confirmed a transition from crystalline into a moderately amorphous structure after amino acid grafting, which made the derivative watersoluble at physiological $\mathrm{pH}$. Composite hydrogels gelated within less than $60 \mathrm{~s}$ when using this WSC together with benzaldehyde terminated 4-arm polyethylene glycol as crosslinker. The compressive modulus of these hydrogels could be easily tuned between $4.0 \pm 1.0 \mathrm{kPa}$ to $31 \pm 2.5 \mathrm{kPa}$, either by changing the crosslinker concentration or total solid content in the final gel. The gels were injectable at the lowest crosslinker as well as total solid content, due to the enhanced elastic behavior. These hydrogel showed biodegradability during a one month incubation period in phosphate buffer saline with weight remaining of $60 \pm 1.5 \%$ and $44 \pm 1.45 \%$ at $\mathrm{pH} 7.4$ and 6.5 , respectively. The cytocompatibility of the gels was tested by fibroblast cell line (i.e. WI-38), which showed good cell viability on the gel surface. Therefore, these hydrogels could be important injectable biomaterial for the delivery purpose in the future.
\end{abstract}

KEYWORDS: biopolymers, chitosan derivative, semisynthetic hydrogels, biodegradable, mechanical strength, self-healing gels 


\section{INTRODUCTION}

Dynamically crosslinked hydrogels are an important class of biomaterials for tissue engineering applications, because of the transient linkages that are reversible and selfhealable. ${ }^{1}$ These hydrogels have the ability to repair their broken network without any external stimuli and maintain the mechanical properties after being damaged. ${ }^{2}$ They possess many similar properties to the native extracellular matrix (ECM), which are critical for 3D cell culture in order to maintain mechanical support, degradation and to enable the appropriate cellular functions. ${ }^{3}$

The physically crosslinked hydrogels are also interesting, owing to the stress relaxation occurs by breaking and subsequent forming of the crosslinked bonds. ${ }^{4}$ Nevertheless, the uncontrolled gelation and degradation time, and the poor tissue dwell time due to the dilution factor followed by dissolution has limited their applications. ${ }^{5}$ On the other hand, the covalently crosslinked hydrogels have high mechanical stability and durability, but the gel formulation might involve toxic catalyst or initiators and also often degrade very slowly., Hence, there is a need for in situ crosslinked dynamic hydrogels without any need for external catalyst, which mimic the living tissues to repair themselves after injury to restore the body function. ${ }^{3,8}$

Chitosan is an amino polysaccharide derived from chitin, which is the main component of crustacean exoskeleton e.g. crabs, shrimp shell etc. and most abundantly available biopolymer after cellulose. It is attracted for biomedical applications, because of several beneficial properties such as low toxicity, biodegradability, antitumor activity and antibacterial properties. However, the low water solubility of the pure chitosan at physiological $\mathrm{pH}$ has limited its application for the use of biomaterial fabrication. ${ }^{9-11}$

In recent years several of its water-soluble derivatives have been prepared, such as quaternized and pegylated, ${ }^{12}$ arginine grafted, ${ }^{11}$ catechol group grafted, ${ }^{10}$ gallic acid grafted ${ }^{13}$ and carboxymethyl chitosan ${ }^{14,15}$ derivatives are among some of the listed one. In all of these derivatives the incorporated moieties could have a plasticizing effect on the main polymer backbone, thus shielding the inter and intra-molecular interactions that could be evidenced from the reduced decomposition and lowered glass transition temperature $\left(\mathrm{T}_{\mathrm{g}}\right)$. This transition from crystalline form to amorphous structure had made them water-soluble even at physiological $\mathrm{pH},{ }^{16-18}$ and they could be easily processed for hydrogel fabrication as well as several other biomedical applications.

The fabrication of chitosan based crosslinked hydrogels, often involved are crosslinkers such as glutaraldehyde (GTA), hexamethylene diisocynate or epoxy-terminate polyethylene glycol 
(PEG), out of which in addition to the in-vivo cytotoxic effect shown by GTA, the latter two exhibit the undesirably prolonged biodegradation rate. ${ }^{19-21}$ Therefore, multi-arm PEG or 4arm-PEG terminated in aldehyde groups could be a useful alternative crosslinker to obtain transparent chitosan hydrogels, owing to the flexible nature, biodegradable esters groups, as well as multiple crosslinking points that results into dynamic Schiff-base linkages with amine groups under ambient conditions. ${ }^{1,22}$ Some injectable gels show quite slow gelation time $(>20$ min), as shown previously by chitosan-hyaluronan hydrogel. ${ }^{23}$ Therefore, fast gelation is an important requisite for injectable in situ forming hydrogels that involves minimal invasive application procedure, behave smart in order to enables tissue ingrowth and remodeling. ${ }^{24,25}$ On the other hand, tuning of the mechanical factors such as shear or compressive forces of the hydrogels, that arises when cells interact with the ECM, had critical impact on cell growth and proliferation, because they could finally regulate the cell fate. ${ }^{26}$

Here we applied a bioinspired approach to prepare water-soluble chitosan derivative and then its composite hydrogels. So far, no one has carried out the grafting of L-glutamic acid onto chitosan, according to our knowledge, to maintain the zwitterion balance charge in the skeleton plus making the derivative water-soluble at physiological $\mathrm{pH}$. Therefore, as the first step, the L-glutamic acid was grafted onto chitosan polymer chain by one step coupling reaction to obtain its water-soluble derivative. In the subsequent step, the composite hydrogels were fabricated from the chitosan derivative and benzaldehyde terminated 4-arm PEG using Schiff-base linkages between their amine and aldehyde groups, respectively. The obtained chitosan derivative and its hydrogels were characterized by different physicochemical techniques, in order to understand the structure-property relationship, as well as the biodegradability, self-healing ability and the biocompatibility of the newly designed biomaterial.

\section{MATERIALS AND METHODS}

\section{Materials}

All materials were purchased from Sigma-Aldrich unless otherwise stated, Chitosan $(M w=$ 50-190 kDa, degree of deacetylation $(D D)=\sim 80 \%$, L-glutamic acid ( $\geq 99 \%)$, N-(3dimethylaminopropyl)-N'-ethylcarbodiimide hydrochloride (EDC) ( $\geq 99 \%)$, 4-formyl benzoic acid (97\%). The 4-arm polyethylene glycol (4-arm PEG) $(95 \%, M w=10 \mathrm{kDa})$ was purchased from JenKem Technology USA.

\section{Methods}

Preparation of Water Soluble Chitosan. Chitosan (1mM, $163 \mathrm{mg}$ ) was dissolved in phosphate buffer saline (PBS) at $\mathrm{pH}=7.4(0.5 \%$ solution $)$ and soaked overnight. Next day 
the L-glutamic acid ( $2 \mathrm{mM}, 294 \mathrm{mg}$ ) was added to the chitosan solutions, ultrasonicated and stirred for an additional $1 \mathrm{~h}$. This made the $\mathrm{pH}$ of the solution more acidic (i.e. $\mathrm{pH}$ dropped to almost 3.8), which also ensured the complete solubility of chitosan. The $\mathrm{pH}$ of the mixture was slowly raised and adjusted between 5.0-5.5 with 0.5 M NaOH solution. Equimolar EDC to L-glutamic acid (i.e. $2 \mathrm{mM}, 384 \mathrm{mg}$ ) was added to the above solution and the mixture was stirred for $24 \mathrm{~h}$. After the reaction, the solution was dialyzed for 2 days against deionized (DI) water, $5 \mathrm{~h}$ against PBS and $6 \mathrm{~h}$ against DI water. The sample was lyophilized to get the purified polymer denoted as chitosan-g-L-glutamic acid (chit-glu). (Scheme 1).

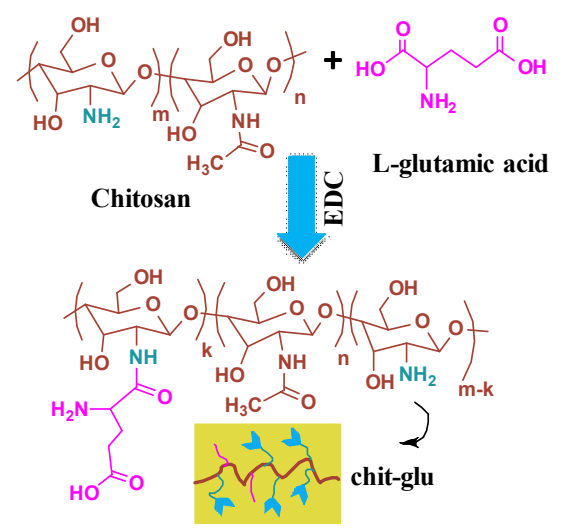

Scheme 1: Preparation of water soluble chitosan (i.e. chit-glu) by EDC coupling reaction.

i. Preparation of aldehyde terminated 4-arm PEG: 4-arm-PEG (0.333 mM, $333 \mathrm{mg})$, 4formylbenzoic acid (3.33 mM, $449.5 \mathrm{mg})$, EDC (4.99 mM, $767 \mathrm{mg}$ ) were separately dissolved in anhydrous chloroform and then the mixture was reacted under inert gas (nitrogen) atmosphere for $48 \mathrm{~h}$. The sample was precipitated in excess diethyl ether and further purified by dialysis for $48 \mathrm{~h}$ and lyophilized, henceforth denoted as PEG-BA (Scheme 2).

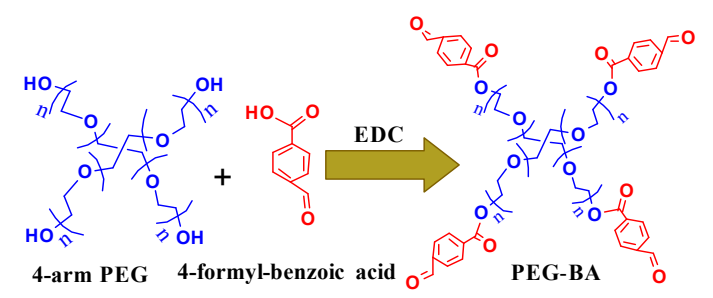

Scheme 2: Preparation of the benzaldehyde terminated star PEG (i.e. PEG-BA).

ii. Fabrication of Hydrogels: The hydrogel preparation was carried out in two series:

a) Keeping chit-glu amount constant and varying PEG-BA: $1 \%$ chit-glu and $20 \%$ PEGBA stock solutions were prepared in distilled water and the crosslinker (PEG-BA) amount was varied i.e. chit-glu/PEG-BA ratio $(\mathrm{R})(\mathrm{w} / \mathrm{w})=1: 2,1: 1,1: 0.5,1: 0.333$. 
b) Total solid content (T) was varied $(2.5 \%, 2.0 \%, 1.5 \%, 1.0 \%)$, while keeping the chitglu/PEG-BA ratios constant (i.e. $\mathrm{R}=1: 0.5$ ).

Both the chit-glu polymer and crosslinker (PEG-BA) were separately dissolved in DI water in a glass vial to get the homogeneous solution. For each desired composition, the specific amount of PEG-BA crosslinker solution was added using micro-pipette into the chit-glu polymer solution and then vortexed for $15 \mathrm{~s}$, which quickly gelated within $60 \mathrm{~s}$, as observed from tilting the vial (Scheme 3).

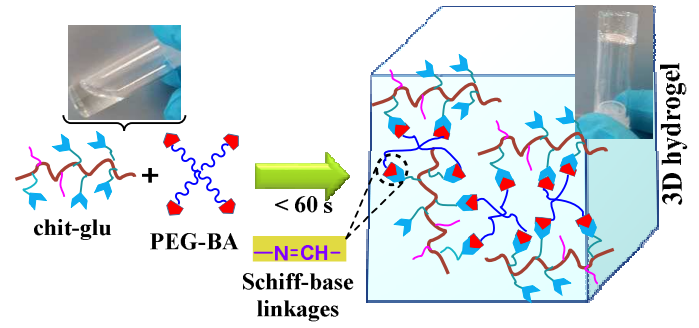

Scheme 3: Preparation of 3D crosslinked hydrogels from modified chitosan (chit-glu) and PEG-BA crosslinker through Schiff-base linkages.

\section{Characterization}

A) Degree of Substitution. The degree of substitution (DS) of the chitosan was determined by potentiometric titration method. ${ }^{27,28}$ Briefly, specific amount of chitosan and chitosan derivatives were dissolved in standard $\mathrm{HCl}$ solution $(0.1 \mathrm{~N})$ and then titrated against standard $\mathrm{NaOH}$ solution $(0.1 \mathrm{~N})$. The amount of $\mathrm{NaOH}$ consumed as a function of $\mathrm{pH}$ was noted continuously and the DS was calculated using the following equation;

$$
A=\frac{\left(V_{2}-V_{1}\right) c}{m} ; D S=\frac{0.203 A}{1-0.153 A}
$$

Where $V_{1}$ is the first end point of excess $\mathrm{HCl}$ used, $V_{2}$ the second end point for $\mathrm{NH}_{2} \cdot \mathrm{HCl}$ deprotonation, $c$ is the concentration of $\mathrm{NaOH}$ used $(0.1 \mathrm{~N}), m$ is the mass of the sample in grams.

B) Proton Nuclear Magnetic Resonance Spectroscopy. For proton nuclear magnetic resonance spectroscopy $\left({ }^{1} \mathrm{H}\right.$ NMR) analysis chit-glu was dissolved in $\mathrm{D}_{2} \mathrm{O}(10$ $\mathrm{mg} / \mathrm{mL})$, pure chitosan $(10 \mathrm{mg} / \mathrm{mL})$ in $1.5 \% \mathrm{D}_{2} \mathrm{O}$ solution of $\mathrm{CD}_{3} \mathrm{COOD}$. PEG and PEG-BA was dissolved in $\mathrm{CDCl}_{3}(15 \mathrm{mg} / \mathrm{mL})$. The NMR spectra were recorded on Varian Mercury $300 \mathrm{MHz}$ Spectrometer using TMS as the internal standard.

C) Fourier Transform Infrared Spectroscopy. Fourier Transform Infrared Spectroscopy (FTIR) measurements was made with Bruker optics tensor 27 using 
attenuated total reflectance (ATR) mode, between $650-3500 \mathrm{~cm}^{-1}$, using 16 scans and a resolution of $4 \mathrm{~cm}^{-1}$.

D) Differential Scanning Calorimetry. The differential scanning calorimetry (DSC) analysis was carried out on NETZSCH DSC 204F1 Phoenix instrument with an automatic sampler changer. The sample was taken and pre-weighed in $100 \mu \mathrm{L}$ Al pan with pierced lid and heated twice under an inert gas $\left(\mathrm{N}_{2}\right)$ atmosphere from $25-220{ }^{\circ} \mathrm{C}$ with the heating rate of $10{ }^{\circ} \mathrm{C} / \mathrm{min}$ and cooled down at the rate of $40{ }^{\circ} \mathrm{C} / \mathrm{min}$ in between the heatings. In the final cycle the sample was heated from $25-350{ }^{\circ} \mathrm{C}$.

E) X-ray Diffraction Analysis. The X-ray diffraction (XRD) patterns were obtained on a PANalytical (Empyrean) diffractometer with Ni-filtered $\mathrm{K} \alpha$ radiation $(\lambda=0.56 \AA)$. A chitosan powder sample was used (50-100 mg), while the WSC and hydrogel samples were casted as thin film $(\sim 1 \mathrm{~mm})$.

\section{Characterization of Hydrogels}

A) Mechanical Testing. Hydrogel discs of different compositions (as mentioned in step (a) and (b)) were fabricated, with sample volumes $=500 \mu \mathrm{L}$ (cross-section diameter $(\mathrm{D})=8.0 \pm 1 \mathrm{~mm}$ and height $(\mathrm{H})=7.0 \pm 1 \mathrm{~mm}$ ) in a plastic syringe using micropipette. The gel samples were covered with paraffin film and used as prepared without storing. The $\mathrm{D}$ and $\mathrm{H}$ of the samples were measured with a digital Vernier caliper. Compression testing was done with a Bose BioDynamic ElectroForce Instrument 5100 using WinTest 4.1 software (TA Instruments, USA). The number of parallel compression samples $(n)$ was more than three $(n>3)$ and the results were averaged \pm SD. The testing was carried out as uniaxial, unconfined compression in air at ambient pressure and temperature. The sample was placed between two compression pistons covered with paraffin film and prevented from sliding with wet cellulose paper (Scheme 4). The compression was carried out with a speed of $10 \mathrm{~mm} / \mathrm{min}$ and up to $65 \%$ of the original sample height. From the results the stress $(\mathrm{kPa})$ was calculated as a force per unit area and the strain $(\mathrm{mm} / \mathrm{mm})$ as a displacement per unit height. The slope of the linear region of stress versus strain curve (i.e. $15-35 \%$ strain), yields the compression modulus. 


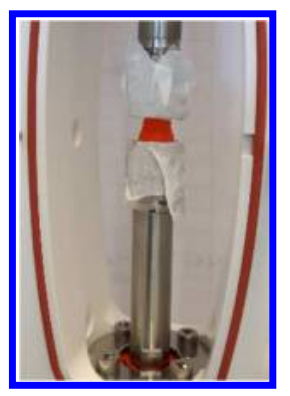

Scheme 4: The hydrogel sample (red) placed between the tips of Bose Compression Testing Machine.

B) In Vitro Degradation. For degradation testing, the hydrogel sample (chit-glu/PEG$\mathrm{BA}=2: 1, \mathrm{~T}=2.5 \%$ ) with size i.e. diameter $=12 \pm 0.5 \mathrm{~mm}$ and height $=10 \pm 0.4 \mathrm{~mm}$ was prepared, washed three times with DI water and freeze-dried. The samples were then incubated in PBS at two different pH i.e. 7.4 and 6.5, for thirty days, with fresh buffer being exchanged after every $3^{\text {rd }}$ day. The degradation rate was determined as weight remaining (ratio \%), ${ }^{29}$ as given by the following equation;

$$
\text { Weight remaining }(\%)=W_{a} / W_{b} \times 100
$$

where $W_{a}$ is the dry gel weight after degradation at different time intervals and $W_{b}$ is the dry gel weight before the start of degradation experiment.

C) Stability Testing. For stability testing the hydrogel components was first sterilized under UV lamp for $30 \mathrm{~min}$ in a clean cell culture lab. The hydrogel samples (vol. 500 $\mu \mathrm{L}$ ) was prepared according to method (b) i.e. by varying the total solid content (T \%), while keeping $\mathrm{R}$ value constant (i.e. chit-glu/PEG-BA = 1:0.5). The samples were prepared in triplicate and immersed in culture media $(400 \mu \mathrm{L})$. The samples were incubated for 7 days in a cell culture incubator under physiological conditions, with fresh media being exchanged after $3^{\text {rd }}$ and $7^{\text {th }}$ days. The $\mathrm{pH}$ of the media was also monitored at aforementioned periods.

D) Self-Healing Experiment. For the self-healing testing two gel samples ( $T=1 \%, R=$ 1:0.5), one original and another stained red with eusine-y were prepared in $5 \mathrm{~mL}$ plastic syringe mold. After preparation, both gels were taken out and transfered into another same size separate syringes. They were then injected through a 22-gauge needle into another syringe mold successively. The injected gels were put intact for $1 \mathrm{~h}$ in air and then incubated in phosphate buffer solution (PBS) for $2 \mathrm{~h}$ at $\mathrm{pH} 7.4$ to obtain the final self-healed gel.

E) In Vitro Cell Viability Assay. Human lung fibroblast cell line WI-38 (from ECACC, Public Health England, UK) was cultured with Dulbecco's Modified Eagle Medium/Ham's Nutrient Mixture F-12 (DMEM/F-12 1:1; Thermo Fisher Scientific) 
supplemented with 10\% fetal bovine serum (South American origin, Biosera, Finland) and $0.5 \%$ Penicillin/Streptomycin $100 \mathrm{U} / \mathrm{mL}$ (P/S; Thermo Fisher Scientific). During biocompatibility testing the cells were trypsinized, counted and plated on top of the hydrogel at density $20000-30000$ cells $/ \mathrm{cm}^{2}$ for three days. For viability analysis, the cultures were stained using a LIVE/DEAD ${ }^{\circledR}$ cell viability/cytotoxicity assay kit (Molecular probes, Thermo Fisher Scientific). In the staining assay Calcein-AM (0.2 $\left.\mu \mathrm{M}, \lambda^{\text {emission }}=488 \mathrm{~nm}\right)$ stains live, intact cells green and Ethydium homodimer-1 (0.8 $\mu \mathrm{M}, \lambda^{\text {excitation }}=568 \mathrm{~nm}$ ) stains dead cells red. After 30 min incubation, the cells were imaged with an Olympus IX51 inverted fluorescent microscope and an Olympus DP30BW digital camera (Olympus, Finland).

F) Statistical Analysis. All the experiments were done in triplicate (i.e. with $n>3$ ), and the presented results are average \pm standard deviation (SD).

\section{RESULTS AND DISCUSSION}

Preparation and Degree of Substitution. The grafting of L-glutamic acid onto chitosan backbone was carried out conveniently under ambient condition and can be controlled by varying the molar ratios $(\mathrm{R})$ of the feeding reagents (chit/glu/EDC; $\mathrm{R}=1: 1: 1$ and 1:2:2), while for all future characterization including hydrogel fabrication the later composition (i.e. $\mathrm{R}=$ $1: 2: 2$, denoted as chit-glu) was used. The potentiometric titration method was used to determine the degree of substitution (DS) of these derivatives, which is the simplest way recently used for this purpose. ${ }^{30-32}$ The titration curves of chitosan and its two derivatives with two different L-glutamic acid grafted content is shown in (Supporting Information (SI) Figure S1A). In the first derivative of titration curves Figure S1B), two inflection points were observed for all the samples. The first inflection point corresponds to the neutralization of excess amount of $\mathrm{HCl}(0.1 \mathrm{~N})$ used, while the second point indicate the deprotonation of the protonated primary amine groups of chitosan $\left(\mathrm{NH}_{2} \cdot \mathrm{HCl}\right)$. The obtained A values (constant in equation 1) were $4.60,4.10$ and $3.99 \pm 0.01$ for $0 \mathrm{mM}$ (pure chitosan), $1 \mathrm{mM}$ and $2 \mathrm{mM} \mathrm{L}$ glutamic acid treated derivatives, respectively. This indicates a slower, but clear transition from higher towards lower titrant $(0.1 \mathrm{~N} \mathrm{NaOH})$ volume consumed and the zwitterion nature of grafted amino acid. The estimated mean DS values obtained were $13 \%$ and $16 \%$ (with SD $= \pm 1.25 \%$ ) for $1 \mathrm{mM}$ and $2 \mathrm{mM}$ L-glutamic acid, respectively using unmodified chitosan as reference control.

The ${ }^{1} \mathrm{H}$ NMR spectra (Figure S2) of pure PEG showing the characteristic peaks $(\mathrm{a}, 5,6)$ of PEG backbone at $3.6 \mathrm{ppm},{ }^{33}$ while after end group modification with 4-formyl benzoic acid 
indicate additional peaks at $7.9 \mathrm{ppm}$, at $8.2 \mathrm{ppm}$ and at $10.10 \mathrm{ppm}$, assigned to the aromatic carbon $(2,3)$ and aldehyde groups $(1)$, respectively of the benzaldehyde end functionality. ${ }^{34}$ Similarly, in case of chitosan, some new peaks appeared after grafting reaction, at $2.4 \mathrm{ppm}$ and at $4.1 \mathrm{ppm}$, which characterize the methylene $(\beta, \gamma)$ and $(\alpha)$ protons, respectively, of Lglutamic acid. ${ }^{35,36}$ Therefore, the ${ }^{1} \mathrm{H}$ NMR spectra shows the successful grafting of Lglutamic acid onto the chitosan backbone. The DS was also calculated from NMR by dividing the integral values of peaks at $2.4 \mathrm{ppm}$ plus $4.1 \mathrm{ppm}$ by acetyl group protons between 1.90-1.93 multiplied by 5 (i.e. $20 \%$ acetyl groups in original sample), which gives $15 \%$ (i.e. a value which corresponds to $2 \mathrm{mM}$ chit-glu). ${ }^{10}$ This indicates that the DS values measured by both potentiometric and NMR methods were in close agreement with each other.

Fabrication of Composite Hydrogels. The hydrogels preparation was carried out by mixing the homogeneous aqueous solutions of the modified chitosan (chit-glu) and crosslinker (PEGBA) by micropipette and then vortexing the mixture for about $15 \mathrm{~s}$. Several different compositions (see methods) were used in order to get an idea of the variation trend in their modulus and self-healing ability. The in situ gelation was fast (i.e. $<60$ s) for all the gel compositions with (SD $\pm 10 \mathrm{~s}$ ) upon varying either the crosslinker content or the total solid content.

The FTIR spectra of PEG (Figure S3A) after end-group functionalization with 4-formyl benzoic acid showing an additional peak at $1707 \mathrm{~cm}^{-1}$, which is the characteristic band of aldehyde $(\mathrm{C}=\mathrm{O})$ groups, while the intensity at $2817 \mathrm{~cm}^{-1}$ becomes more intense, due to the incorporation of the aromatic ring (-C-H stretching). ${ }^{37}$

The FTIR spectra (Figure S3B) of modified chitosan shows the characteristics peak of carboxyl group of L-glutamic acid at $1670 \mathrm{~cm}^{-1} \cdot 38$ and merged with the peak at $1657 \mathrm{~cm}^{-1}$ of amide (I) $-\mathrm{C}=\mathrm{O}$ stretching. ${ }^{39}$ The peak at $1553 \mathrm{~cm}^{-1}$ clearly becomes intense, which corresponds to the amide (II) bond (-CONH- stretching vibration) formation. ${ }^{40,41}$ The two slightly weak bands for pure chitosan at $1423 \mathrm{~cm}^{-1}$ and $1378 \mathrm{~cm}^{-1}$ merged and converted into a single intense band at $1388 \mathrm{~cm}^{-1}$ and is assigned to the amide III band. ${ }^{42}$

The first heating and cooling cycle $\left(25-220-25^{\circ} \mathrm{C}\right)$ in the DSC thermogram (Figure S4A) indicated an endothermal peaks at $119{ }^{\circ} \mathrm{C}, 114{ }^{\circ} \mathrm{C}$ and $128{ }^{\circ} \mathrm{C}$ for chitosan, chit-glu and chitglu/PEG-BA, respectively, which corresponds to the evaporation of water molecules from the sample. The higher temperature for the hydrogel indicates its hydrophilic nature due to the incorporated PEG, which bound the water molecules strongly in the network structure. The 
same isotherm also shows the glass transition temperature $\left(T_{g}\right)$ at $203{ }^{\circ} \mathrm{C}, 186{ }^{\circ} \mathrm{C}$ and $183{ }^{\circ} \mathrm{C}$ for chitosan, chit-glu polymer and chit-glu/PEG-BA hydrogel, respectively. The same decrease in $T_{g}$ was also observed in the previous report for the chitosan blends. ${ }^{43} \mathrm{~A}$ reverse transition was manifested by the cooling exotherms (Figure SI-4A), upper) also from higher temperature $\left(211^{\circ} \mathrm{C}\right)$ for chitosan and to $203{ }^{\circ} \mathrm{C}$ for chit-glu plus its hydrogel. During the $2^{\text {nd }}$ heating and cooling cycle (Figure S4B) in the same temperature range $\left(25-220^{\circ} \mathrm{C}\right)$ there was no exo/endothermal peaks observed, which indicates that no apparent physical/chemical changes are taking place further in this temperature range.

The samples were finally heated to higher temperature $\left(25-350{ }^{\circ} \mathrm{C}\right)$ (Figure S4C), which showed the characteristic decomposition temperature $\left(T_{d}\right)$ of chitosan at $315{ }^{\circ} \mathrm{C}$ was observed. ${ }^{44,45}$ After the grafting reaction, the $T_{d}$ decreased to $275{ }^{\circ} \mathrm{C}$ for chit-glu and for the hydrogel (chit-glu/PEG-BA) to $273{ }^{\circ} \mathrm{C}$. A similar decrease in the decomposition temperature has been observed also after chitosan modification. ${ }^{46}$ These results indicates that after grafting reaction, the crystalline form of chitosan was transformed into somewhat amorphous structure, which decomposed at a lower temperature than the starting raw material.

The XRD pattern of chitosan shows a characteristic strong peak ${ }^{47}$ at $2 \theta=20^{\circ}$ (Figure 1) (a). After grafting of L-glutamic acid onto chitosan backbone, the sharp amorphous peak (at $20^{\circ}$ (a)) was greatly suppressed and replaced by a broadened weaker peak at $23^{\circ}$ and several characteristic peaks from the crystalline phase (b). The lower intensity of the crystalline peak and the appearance of the characteristic diffraction peaks $(23,25,28)$ (b) shows that the chitosan was partially crystallized after the amino acid grating. The "peak" or the broad hump at around $23^{\circ}$ is from the interaction of $\mathrm{x}$-rays and amorphous material, and the sharp peaks at higher angle (i.e. $28^{\circ}$ and $37^{\circ}$ ) are from the crystalline phases. This clearly indicates that the original structure of chitosan was destroyed and converted into amorphous/crystalline form after the amino acid grating. ${ }^{39,48}$ Therefore we could presume that the carboxyl groups of the grafted L-glutamic acid has developed ionic interaction with the amine groups of chitosan (long range ordered structures) and hence facilitated its water solubility. ${ }^{49,50}$ 


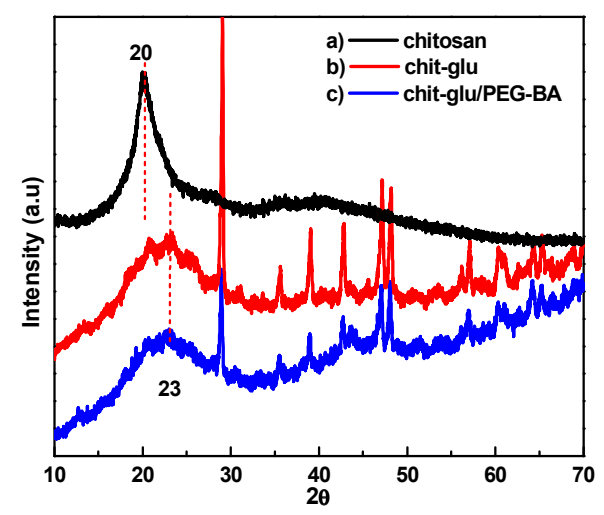

Figure 1: XRD patterns of a) chitosan, b) modified chitosan (chit-glu) and c) hydrogel (chit-glu/PEGBA).

Similarly the hydrogel (chit-glu/PEG-BA), also showing the same broadened peak (at $23^{\circ}$ ) as well as other higher angle crystalline peaks and the presence of amorphous phase(s) within the sample may result in irregular base line with noise (c). While, the minor peak of PEG at $23.4^{\circ}$ was merged with the broadened peak of chit-glu at $23^{\circ}$, which indicated the partial amorphous/crystalline morphology of the chit-glu/PEG-BA gel network. ${ }^{51,52}$

Mechanical Characterization of Hydrogels. For the complete mechanical properties evaluation, two different series of hydrogels were prepared, in order to find the different possible ways for tuning the gel mechanical strength.

a) By Varying the Crosslinker Amount (w/w). In this first series of hydrogels, the polymer ratio was kept constant and the relative crosslinker ratios $(\mathrm{w} / \mathrm{w})$ varied. The four different gel compositions along with their mechanical properties are presented in the Table 1 below.

Table 1: Hydrogels having constant polymer (chit-glu) and various crosslinker (PEG-BA) ratios.

\begin{tabular}{r|c|c|r|c}
\hline Gel type & $\begin{array}{c}\text { chit-glu/PEG-BA } \\
\text { ratio: } \mathrm{R}(\mathrm{w} / \mathrm{w})\end{array}$ & $\begin{array}{c}\text { Fracture stress } \\
(\mathrm{kPa})\end{array}$ & $\begin{array}{c}\text { Fracture strain } \\
(\mathrm{mm} / \mathrm{mm})\end{array}$ & Modulus (kPa) \\
\hline Gel-A & $1: 2.0$ & $18 \pm 1.5$ & $0.47 \pm 0.05$ & $31 \pm 2.5$ \\
\hline Gel-B & $1: 1.0$ & $15 \pm 1.0$ & $0.53 \pm 0.05$ & $15 \pm 2.0$ \\
\hline Gel-C & $1: 0.5$ & $20 \pm 1.5$ & $0.60 \pm 0.05$ & $11 \pm 1.5$ \\
\hline Gel-D & $1: 0.333$ & $\mathrm{e}^{*}$ & $\mathrm{f}^{*}$ & $4.0 \pm 1.0$ \\
\hline
\end{tabular}

Note: $\mathrm{e}^{*}$ and $\mathrm{f}^{*}$ denote that no fracture stress and strain was observed for gel-D, with $\mathrm{n}=6$ for each sample. 
The gels with the above four different compositions were analyzed by compression testing as shown in Figure 2A. The modulus values for each sample was calculated from the slope of the stress $(\mathrm{MPa})$ vs strain $(\mathrm{mm} / \mathrm{mm})$ curves between 15-35 (\%) strain, where they exhibit almost linear (elastic) behavior. The gels behaved stronger when higher amount of crosslinker was used (i.e. $\mathrm{R}=1: 2)$, with increased modulus value $(31 \pm 2.5 \mathrm{kPa})$. On the other hand, decreasing of the relative crosslinker content (i.e. $\mathrm{R}=1: 0.333$ ) softens the hydrogels and shows the lower modulus $(4 \pm 1.0 \mathrm{kPa})$. The same decreasing trend was also observed for the fracture stress and strain values, except in the case of the gel-C $(\mathrm{R}=1: 0.5)$, which showed a sudden increase in the fracture stress. In the case of gel-D there was no fracture observed until $65(\%)$ of strain (Figure 2A), which indicated the complete self-recovery ability. When the gel-D was further compressed three times, it recovered to its original shape $(\mathrm{t}<10 \mathrm{~s})$ as shown in Figure 2B.

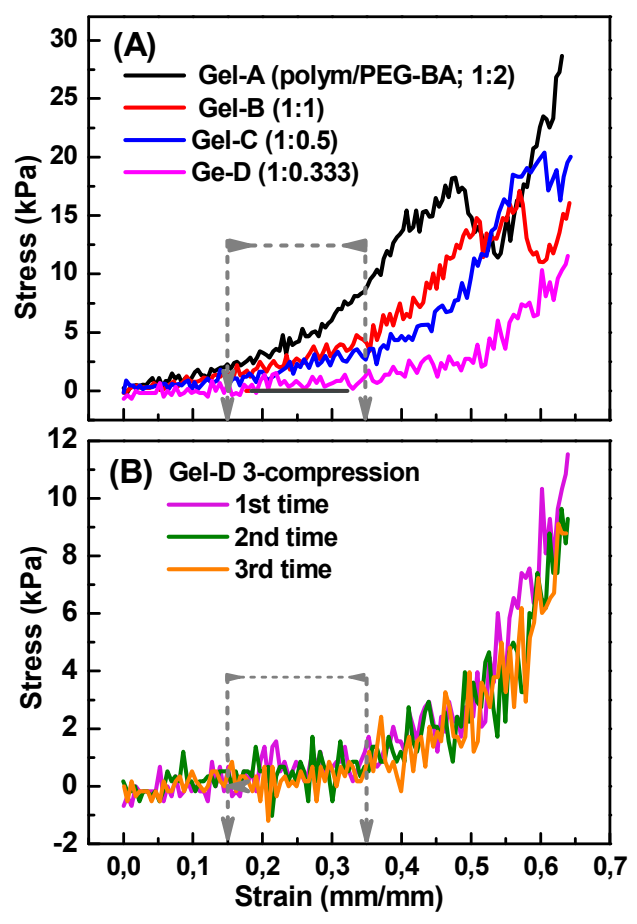

Figure 2: A) Mechanical compression testing of hydrogels with constant polymer (chit-glu) concentration and various amount (w/w) of crosslinker PEG-BA (Gel-A to D), B) Three compression of Gel-D.

The data points from Figure 2A between 15-45\% were plotted and analyzed by linear fitting in order to obtain the $\mathrm{R}^{2}$ values for the different gel compositions (Figure 3 ). For the best fitting results the $\mathrm{R}^{2}$ values for gel-A, gel-B, gel-C were $0.94,0.84,0.86$, while for gel-D the 
$\mathrm{R}^{2}$ was 0.44 even though the fitting was further extend to a higher strain region (18-44\%), which is an indication of the low instrumental sensitivity for the softer gel.

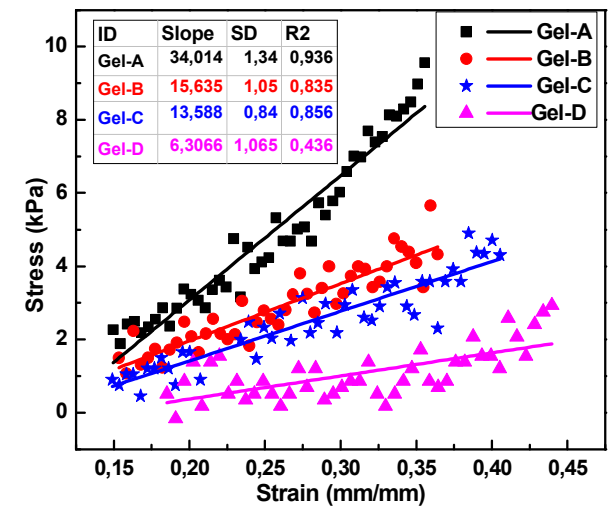

Figure 3: Linear fitting of the actual stress-strain data points (adapted from Figure 2A) to get the Rsquare values for different gel compositions.

The compression and release assay of the gel-D inside the tips of a compression-testing machine is shown in Figure 4, which indicates the self-recovery ability after each compression, at the lowest crosslinker concentration. Hence, the softer gel (gel-D) could be one extremely softer gels in this series, that can be applied as an injectable gel.

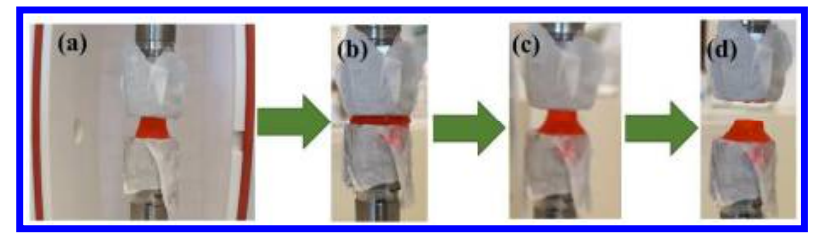

Figure 4: Gel-D compression and release assay a) between the tips, b) compressed, c) released, d) recovered gel after compression.

Note, that any of the gel compositions (i.e. A, B, and C, except gel-D due its too much softness) presented in Table 1, could be further utilized in order to modulate their mechanical properties upon varying the total solid content in the gel. Hence, here we assumed gel-C could be optimal composition based on higher fracture strength and was further explored for mechanical testing by varying the water content (\%).

b) Changing the Total Solid Content. The second series of hydrogels composed of constant polymer/crosslinker $(2: 1)$ ratios and the total solid content $(\mathrm{T} \%)$ in the gel was changed from $1.0 \%-2.5 \%$. The mechanical properties of the resultant gel is given in Table 2.

Table 2: Hydrogels with constant polymer (chit-glu) and crosslinker (PEG-BA) ratios (w/w $=1.0: 0.5)$ and the total solid content in the final gel was varied. 


\begin{tabular}{c|c|r|r|c}
\hline $\begin{array}{c}\text { Gel- } \\
\text { type }\end{array}$ & $\begin{array}{c}\text { Total solid } \\
\text { content }(\mathrm{T} \%)\end{array}$ & $\begin{array}{r}\text { Fracture } \\
\text { stress }(\mathrm{kPa})\end{array}$ & $\begin{array}{r}\text { Fracture strain } \\
(\mathrm{mm} / \mathrm{mm})\end{array}$ & Modulus $(\mathrm{kPa})$ \\
\hline Gel-1 & 2.5 & $52 \pm 2.0$ & $0.47 \pm 0.05$ & $21 \pm 2.5$ \\
\hline Gel-2 & 2.0 & $47 \pm 1.5$ & $0.53 \pm 0.05$ & $16 \pm 2.0$ \\
\hline Gel-3 & 1.5 & $22 \pm 1.0$ & $0.59 \pm 0.03$ & $10 \pm 1.5$ \\
\hline Gel-4 & 1.0 & $\mathrm{~g}^{*}$ & $\mathrm{~h}^{*}$ & $5.0 \pm 1.0$ \\
\hline
\end{tabular}

Note: $\mathrm{g}^{*}$ and $\mathrm{h}^{*}$ denote that no fracture stress and strain was observed for gel-4, with $\mathrm{n}=6$ for all the gels.

The compression testing results of these hydrogel compositions indicated that, with a higher total solid content (i.e. gel-1, $\mathrm{T}=2.5 \%$ ) they behaved harder and showed higher compressive modulus $(21 \pm 2.5 \mathrm{kPa})$ (Figure $5 \mathrm{~A})$. Note that the modulus value was calculated from the slope in the same linear region (15-35\%) of the stress vs strain curve, as was done in the previous section. The gel became softer (i.e. slope declined), when the concentration ( $\mathrm{T}$ $\%)$ was decreased gradually, until gel-4 $(\mathrm{T}=1.0 \%)$, which showed the lowest modulus value (i.e. $5.0 \pm 1.0 \mathrm{kPa}$ ) amongst all the gels. The same lowering trend was also shown by the fracture stress, while the fracture strain was enhanced resulted from the higher elasticity (softness) of the gel. On the other hand, the gel-4 (with more water content i.e. $99 \%$ ) didn't fracture until the maximum compression (65\%) was achieved and showed the self-recovery ability after releasing the pressure. After several compression and release assay $(n=3$ shown here), it can automatically recover $(\mathrm{t}<30 \mathrm{~s})$ to its original shape (Figure $5 \mathrm{~B}$ ). 

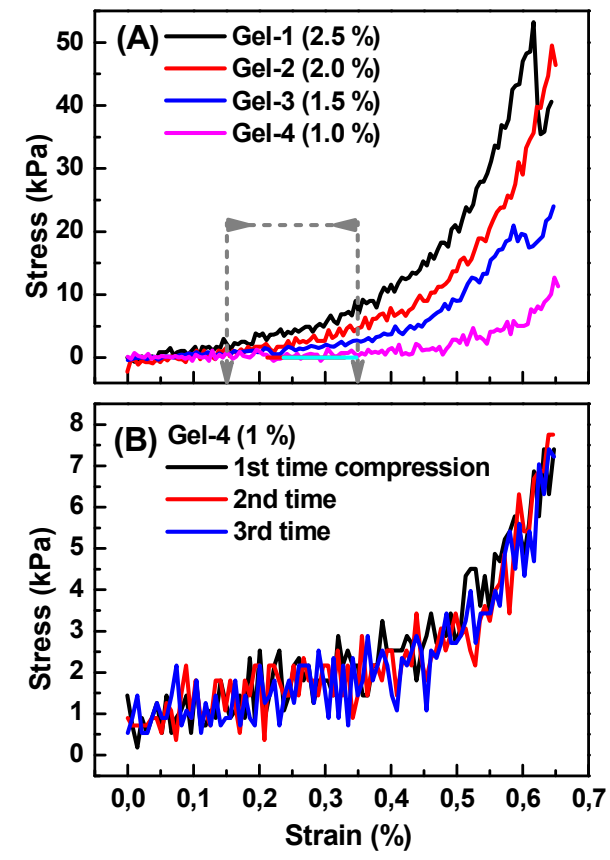

Figure 5: Compression testing of hydrogels, A) with constant polymer/crosslinker ratios $(w / w=2: 1)$ and various total solid content ( $\mathrm{T} \%$ ) in the final gel, B) Three consecutive compressions of gel-4. The mechanical behavior of these gels as a function of polymer/crosslinker ratios (R), as well as with the varying total solid content ( $\mathrm{T} \%$ ), could be further simplified as illustrated in Figure 6 . The specific optimum polymer/crosslinker (2:1) ratio of gel-C is highlighted by dotted circle, that was further extended to see the effect of varying $\mathrm{T} \%$ on modulus value. Note, that the variation in the two compositions simultaneously also indicates a cross-over point, where the two gels (gel-C and gel-3) almost matches in mechanical behavior, with a modulus value of $10 \pm 1.0 \mathrm{kPa}$ and fracture stress of $21 \pm 1.0 \mathrm{kPa}$ (see Tables $1 \& 2$ values ). The similar mechanical properties of the two gels could be clearly attributed to almost the same $\mathrm{T} \%$ as well as similar $\mathrm{R}$ values for both the gels. 
Figure 6: Effect of polymer/crosslinker (w/w) ratios (black-bottom/left axis) and total solid content ( $\mathrm{T} \%$ ) (deep pink-top/right axis) on the compressive modulus of hydrogels.

Hence, the modulus of these gel systems can be easily tuned based on variation of the two main experimental parameters, that is the crosslinker amount $(\mathrm{w} / \mathrm{w})$ and the total solid content ( $\mathrm{T} \%$ ), in order to get the specific hydrogels that could mimic the mechanical strength of the particular tissue. For example, gel-3 might be an appropriate candidate for neural tissue engineering, owing to the similarity in the compressive strength properties i.e. the modulus matches to that of the rabbit brain (i.e. $10 \mathrm{kPa}$ ). ${ }^{53}$ The short gelation time is also important factor for designing the injectable hydrogels, such as our gel system has quick gelation time (i.e. $\mathrm{t}<60 \mathrm{~s}$ ), which is advantageous than the previously prepared injectable hydrogels from water soluble chitosan and aldehyde group modified hyaluronic acid, which showed a compressive modulus of $10-30 \mathrm{kPa}$, but had a longer gelation time i.e. 1-4 min. ${ }^{54}$ Similarly, the compressive modulus of another such kind of injectable composite photo-crosslinked hydrogels of methacrylated glycol chitosan and hyaluronic acid was tuned from 0.7 to $17 \mathrm{kPa}$ as a function of irradiation time from $40-600 \mathrm{~s}^{55}$ These results strongly indicates that modulating the mechanical factors plays a key role in determining the end use of these hydrogel materials. Note, that several mechanical characterization techniques had been used in the literature to deduce the modulus of the hydrogels, but it is important to compare the modulus values of the compression testing only here. For instance, several soft living tissues, such as human fibrotic liver, breast tumor and thyroid tissues shows a compression modulus of $1.6 \mathrm{kPa}, 4 \mathrm{kPa}$ and $9 \mathrm{kPa}$ respectively. ${ }^{56}$

Injectability and Self-Healing Experiment. In order to study the dynamic nature and the true self-healing ability of these hydrogels, they were further subjected to injectability testing. For this purpose, the two gelated hydrogel discs (Figure 7 (a)) one as original and another 
colored as red were taken into $5 \mathrm{~mL}$ plastic syringes (b). The Schiff-base linkages (i.e. the reversible chemical bonds between the amine groups of chit-glu and the aldehyde groups of PEG-BA) in the as prepared hydrogels is shown in Figure $7(\mathrm{X})$. The two gels were then injected through a 22-gauge needle into another same size syringe mold (c, d), successively. After the injection, the gel network (i.e. Schiff-base linkages) were dissociated reversibly into parent amine $\left(-\mathrm{NH}_{2}\right)$ and aldehyde (-CHO) groups of chit-glu and PEG-BA, respectively, as shown in the form of broken network (Figure 7 (Y)). For the self-healing process to achieve, the injected gel samples were first kept untouched in air for $1 \mathrm{~h} \mathrm{(e)} \mathrm{and}$ then incubated in PBS ( $\mathrm{pH}=7.4$ ) for $2 \mathrm{~h}(\mathrm{f})$. Finally, the gel was taken out from the mold ( $\mathrm{g}$ ), which showed the complete self-healed gel network structure (Figure $7(Z)$ ). The same gel was then lifted up with a plastic tweezer several times as well as from different positions (h, i) and behaved as free standing gel. This indicated that it was self-healed reversibly (i.e. the Schiff-base linkages were reformed). Therefore, these kinds of transparent self-healing gels are important class of materials for the fabrication of injectable biomaterials for the delivery purposes in the future.

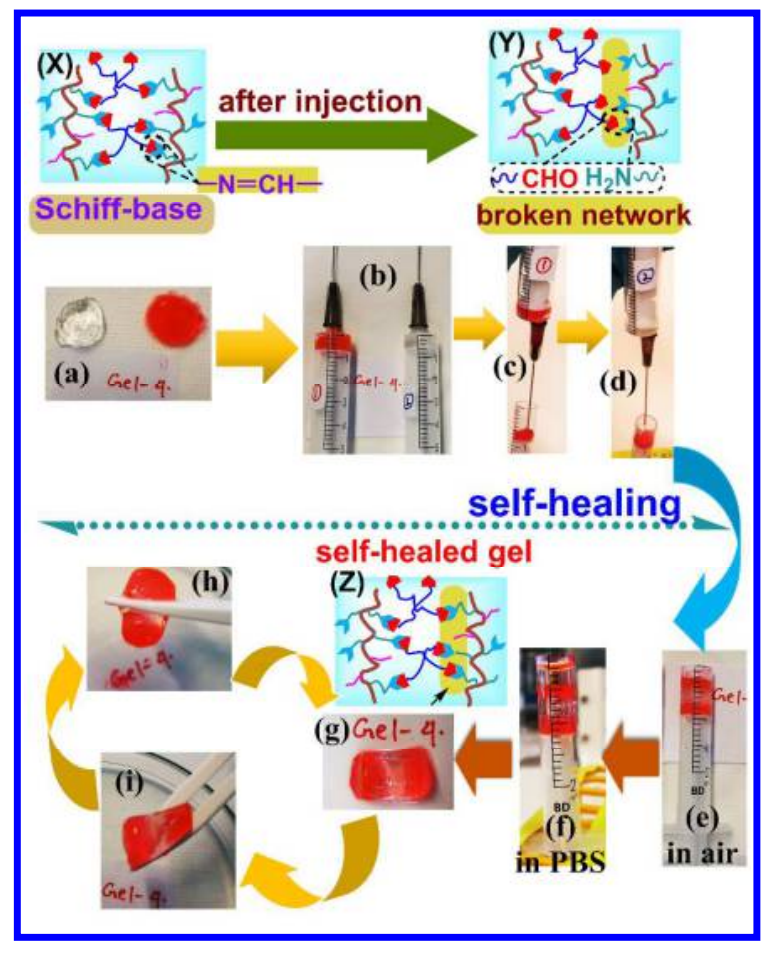

Figure 7: Injectability and self-healing experiment of chit-glu/PEG-BA hydrogels: (a) as prepared hydrogel discs ((X) gel network showing Schiff-base linkages), (b) gels were taken into syringes, (c, d) gels were injected through 22-gauge needle into syringe-mold successively ((Y) broken gel 
network), (e) $1 \mathrm{~h}$ in air, (f) $2 \mathrm{~h}$ in PBS (pH = 7.4), (g) self-healed gel, (h, i) self-healed gel were lifted from different positions ((Z) self-healed gel network).

The self-healed gels in the same way were then subjected to mechanical compression testing (Figure 8), in order to investigate the difference in mechanical behavior before and after the healing process. The gels showed almost the same slope during three compressions and release assays, which provided further evidence of the dynamic/reversible nature of the Schiff-base linkages.

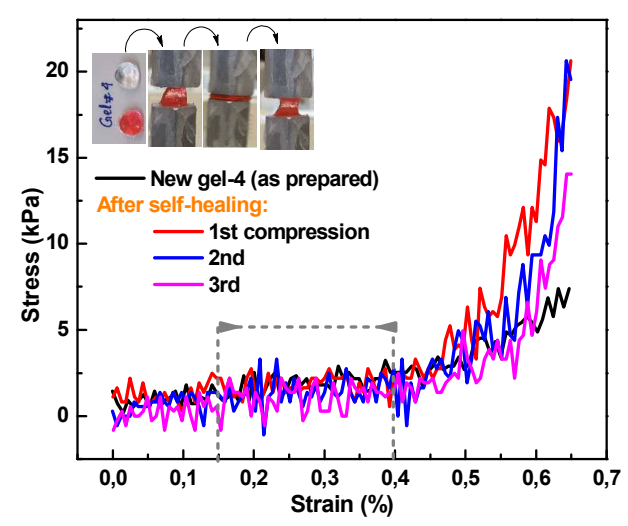

Figure 8: Mechanical compression of the self-healed hydrogel (gel-4) after injection into the mold. Three successive compression and release assays of the same gel.

This indicates that the self-healing properties of such soft hydrogel materials can also be investigated by compression testing using repeated compression and release assays. Therefore, the hydrogels with self-healing ability can recover the viscoelastic properties of the network due to the reversible nature of such linkages. ${ }^{57}$

In Vitro Degradation Testing. The degradation rate (\% weight remaining) of the hydrogels (chit-glu/PEG-BA $2.5 \%$ ) was studied at two different $\mathrm{pH}$ i.e. $\mathrm{pH} 6.5$ and 7.4. The weight remaining (\% ratios) as a function of incubation times (days) at two $\mathrm{pH}$ is shown in Figure 9 (left axis). The hydrogels shows $78 \pm 1.0 \%$ and $63 \pm 1.0 \%$ weight remaining after five days incubation, at $\mathrm{pH} 7.4$ and 6.5, respectively. After thirty days the weight remaining was $60 \pm$ $1.5 \%$ and $44 \pm 1.45 \%$ for the gel incubated at $\mathrm{pH} 7.4$ and 6.5 , respectively. The study at two different $\mathrm{pH}$ clearly indicates that these hydrogel degraded faster under acidic conditions than under the physiological $\mathrm{pH}$. 


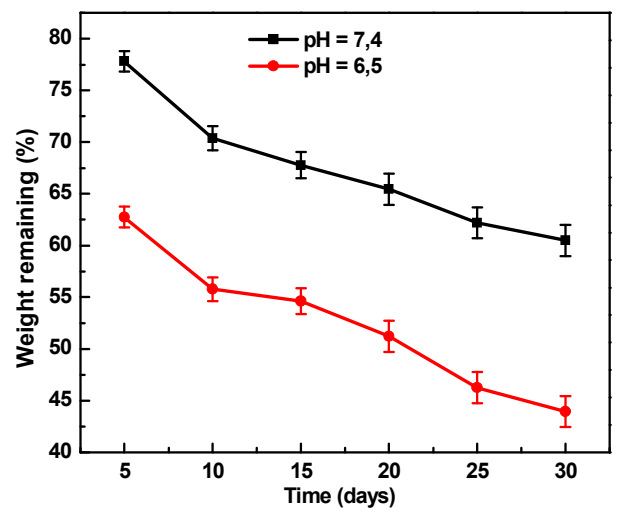

Figure 9: Degradation plot of chit-glu/PEG-BA (2.5\%) hydrogels in physiological buffer $(\mathrm{pH}=7.4)$ and acidic buffer $(\mathrm{pH}=6.5)$ for 30 days.

Note, that the gels incubated at $\mathrm{pH}=7.4$ became rigid, while the gels degraded in acidic buffer (i.e. $\mathrm{pH}=6.5$ ) were unstable to be lifted after incubation in pure water. The attribution can be made to the close $\mathrm{pH}=6.5$ to the $\mathrm{pK}_{\mathrm{a}}$ value of chitosan (6.2-7.0), at which high level of free amine groups of chitosan were protonated and rarely available for Schiff-base linkages thus making the gels weaker and unstable at the end..$^{58,59}$

Stability Testing in Cell Culture Media. The stability of the hydrogels with various T (\%) was studied in cell culture media for 7 days (Figure 10), with media exchanged after every $3^{\text {rd }}$ day. The $\mathrm{pH}$ of the medium was also monitored after $3^{\text {rd }}$ and $7^{\text {th }}$ days (Figure 10), showing that upon diluting the gel sample from $2.5 \%$ to $1.0 \%$, the $\mathrm{pH}$ of the medium was shifted slightly towards alkaline region $(\mathrm{pH} \sim 7.7 \pm 0.1)$ after $7^{\text {th }}$ day.

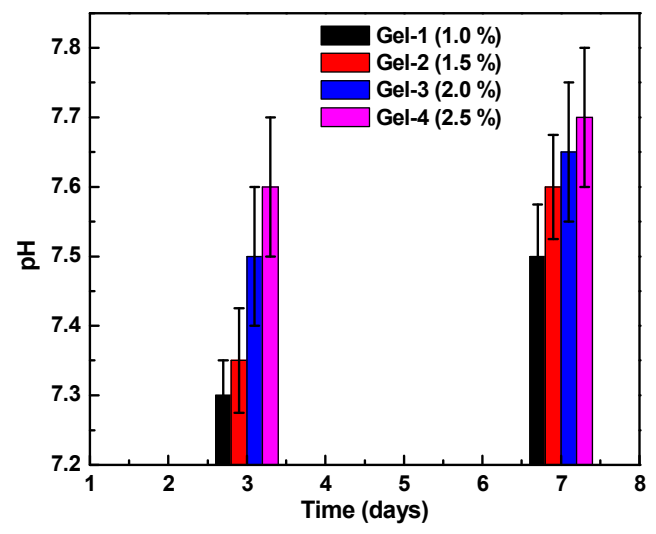

Figure 10: Stability testing of chit-glu/PEG-BA hydrogels with different (T \%) in cell culture media for 3 days and 7 days. 
In Vitro Cell Viability Assay. The in vitro cell viability assay is a promising strategy in recent years for testing the cytocompability of the newly designed biomaterials. ${ }^{60,61}$ Therefore, the biocompatibility of these hydrogels was primarily tested by human fibroblast cell lines (WI-38) cultured for one day and three days on the top of hydrogels (Figure 11). The live and dead cells were stained as green and red, respectively. During the day one culture time most of the cells were dead on the pure chitosan hydrogels (Figure 11A) as compared to chit-glu/PEG-BA hydrogel (B). Similarly after $3^{\text {rd }}$ day culture time, there were rarely cell adhered to the pure chitosan hydrogel (C) and the gel also showed slight degradation. On the other hand, the chit-glu/PEG-BA hydrogel (D) shows mostly live cells and they were also elongated, which can be more apparent from the enlarged image (d) (scale bar $200 \mu \mathrm{m})$. . Moreover, at the bottom of the same $3^{\text {rd }}$ day well of the chit-glu/PEG-BA gel (E) more elongated and also proliferated cells were observed. The same image was further magnified to clearly see the cell shape and morphology (e) (scale bar $200 \mu \mathrm{m}$ ). We can see that this hydrogel is safe for the cells, as they grow quite nicely in the gel microenvironment. Hence, the results obviously indicate that these self-healable hydrogels could be good materials for fabricating injectable biomaterial for the delivery application in the future.
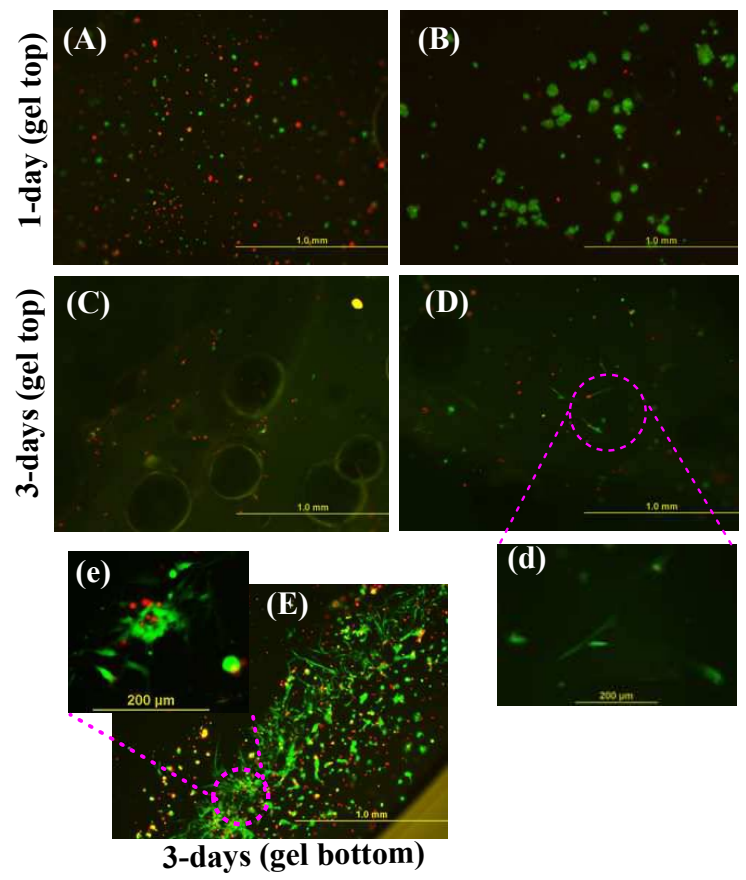

Figure 11: Fibroblast cell line (i.e. WI-38) cultured (green and red stands for live and dead cells, respectively) for one day: (A) on chitosan hydrogel, (B) on chit-glu/PEG-BA hydrogel. For three 
days: (C) on chitosan hydrogel, (D) on chit-glu/PEG-BA hydrogel, d) enlarged cells and (E) in the bottom of the same 3 days well (of chit-glu/PEG-BA hydrogel), e) enlarged cells. Scale bar: (A-E) = $100 \mu \mathrm{m}$ and $(\mathrm{d}, \mathrm{e})=200 \mu \mathrm{m}$.

\section{CONCLUSIONS}

The water soluble chitosan is promising biomaterials for hydrogel fabrication due to its low cost, easy processing and good biocompatibility. Taking the advantage of the anionic charged amino acid, L-glutamic acid, which transferred into zwitterion form after being grafted onto chitosan, retaining the overall charge unperturbed and resulted into a water-soluble chitosan derivative. The grafted amino acid has greatly suppressed the inter- and intramolecular interaction between chitosan chains, thus reducing its crystallinity and facilitating water solubility. This water soluble chitosan derivative could be processed for application, such as for hydrogel fabrication with benzaldehyde terminated 4-arm branched PEG as crosslinker, resulting into semisynthetic or composite dynamic hydrogels. The Schiff-base linkages in these hydrogels originated from the amine group of chitosan and aldehyde groups of 4-arm PEG, are reversible, making the hydrogel self-healable. The mechanical properties of these hydrogels could be easily tuned by either varying the crosslinker concentration or the total solid content in the hydrogels. The hydrogels showed injectable and self-healing properties, re-crosslinking after heavy manipulation and returning to intact gel state during incubation. Moreover, the gels show biodegradability in PBS at pH 7.4 and 6.5, as tested for one month period. Therefore, owing to the injectable, self-healing and biocompatible nature, these hydrogels could be a promising candidate for the delivery purpose in the future.

\section{ACKNOWLEDGMENTS}

We thanks, the Tampere University of Technology (internal funding for postdoctoral researchers) and TEKES - Finnish Funding Agency for Innovation project Human Spare Parts for financially supporting this work.

\section{Notes}

The authors declare that they have no conflict of interest.

\section{ASSOCIATED CONTENT}

\section{Supporting Information}

The Supporting Information is available free of charge on the ACS Publications website: Potentiometric titration curve, ${ }^{1}$ NMR and FTIR spectra, DSC thermogram.

\section{AUTHOR INFORMATION}

Corresponding authors: 
musammir_khan@yahoo.com $\left({ }^{\dagger,}\right\lrcorner$ M. Khan), minna.kellomaki@tut.fi $\left({ }^{\dagger,}{ }^{\prime}\right.$ M. Kellomaki)

\section{REFERENCES}

(1) Zhang, Y.; Tao, L.; Li, S.; Wei, Y. Synthesis of Multiresponsive and Dynamic Chitosan-Based Hydrogels for Controlled Release of Bioactive Molecules. Biomacromolecules 2011, 12, 2894-2901.

(2) Huang, W.; Wang, Y.; Chen, Y.; Zhao, Y.; Zhang, Q.; Zheng, X.; Chen, L.; Zhang, L. Strong and Rapidly Self-Healing Hydrogels: Potential Hemostatic Materials. $\underline{A d v}$. Healthc. Mater. 2016, 5, 2813-2822.

(3) Hoare, T. R.; Kohane, D. S. Hydrogels in Drug Delivery: Progress and Challenges. Polvm. 2008, 49 1993-2007.

(4) Zhao, X.; Huebsch, N.; Mooney, D. J.; Suo, Z. Stress-Relaxation Behavior in Gels with Ionic and Covalent Crosslinks. L Appl. Phvs. 2010, 107, 063509.

(5) Shih, H.; Lin, C. C. Cross-Linking and Degradation of Step-Growth Hydrogels Formed by Thiol-Ene Photoclick Chemistry. Biomacromolecules 2012, 13, 20032012.

(6) Chang, R.; Wang, X.; Li, X.; An, H.; Qin, J. Self-Activated Healable Hydrogels with Reversible Temperature Responsiveness. ACS Appl. Mater. Interfaces 2016, 8, 25544-25551.

(7) Hacker, M. C.; Nawaz, H. A. Multi-Functional Macromers for Hydrogel Design in Biomedical Engineering and Regenerative Medicine. Int.J. Mol.S.Si. 2015, 16, 27677-27706.

(8) Kharkar, P. M.; Kiick, K. L.; Kloxin A. M. Designing Degradable Hydrogels for Orthogonal Control of Cell Microenvironments. Chem. Soc. Rev. 2013, 42, 7335 7372.

(9) Ding, B.; Gao, H.; Song, J.; Li, Y.; Zhang, L.; Cao, X.; Xu, M.; Cai, J. Tough and Cell-Compatible Chitosan Physical Hydrogels for Mouse Bone Mesenchymal Stem Cells in Vitro. ACS Appl. Mater. Interfaces 2016, 8, 19739-19746.

(10) Kim, K.; Ryu, J. H.; Lee, D. Y.; Lee, H. Bio-Inspired Catechol Conjugation Converts Water-Insoluble Chitosan into a Highly Water-Soluble, Adhesive Chitosan Derivative for Hydrogels and LbL Assembly. Biomater. Sci. 2013, 1, 783-790.

(11) Xiao, B.; Wan, Y.; Zhao, M.; Liu, Y.; Zhang, S. Preparation and Characterization of Antimicrobial Chitosan-N-Arginine with Different Degrees of Substitution. Carbohydr. Polvm. 2011, 83, 144-150. 
(12) Mao, S.; Shuai, X.; Unger, F.; Wittmar, M.; Xie, X.; Kissel, T. Synthesis, Characterization and Cytotoxicity of Poly(ethylene glycol)-Graft-Trimethyl Chitosan Block Copolymers. Biomaterials 2005, 26, 6343-6356.

(13) $\mathrm{Hu}$, Q.; Wang, T.; Zhou, M.; Xue, J.; Luo, Y. In Vitro Antioxidant-Activity Evaluation of Gallic-Acid-Grafted Chitosan Conjugate Synthesized by Free-RadicalInduced Grafting Method. J.Agric. Food Chem. 2016, 64, 5893-5900.

(14) Mourya, V. K.; Inamdar, N. N.; Tiwari, A. Carboxymethyl Chitosan and Its Applications. Adv. Mat. Lett. 2010, 1, 11-33.

(15) De Abreu, F. R.; Campana-Filho, S. P. Characteristics and Properties of Carboxymethylchitosan. Carbohvdr. Polvm. 2009, 75, 214-221.

(16) Kumirska, J.; Czerwicka, M.; Kaczynski, Z.; Bychowska, A.; Brzozowski, K.; Thoming, J.; Stepnowski, P. Application of Spectroscopic Methods for Structural Analysis of Chitin and Chitosan. Mar. Drugs 2010, 8, 1567-1636.

(17) Cervera, M. F.; Heinämäki, J.; Krogars, K.; Jörgensen, A. C.; Karjalainen, M.; Colarte, A. I.; Yliruusi, J. Solid-State and Mechanical Properties of Aqueous Chitosan-Amylose Starch Films Plasticized With Polyols AAPS PharmSciTech 2004, $5,109-115$.

(18) Matveev, Y. I.; Grinberg, V. Y.; Tolstoguzov, V. B. The Plasticizing Effect of Water on Proteins, Polysaccharides and Their Mixtures. Glassy State of Biopolymers, Food and Seeds. Food Hvdrocoll. 2000, 14, 425-437.

(19) Poursamar, S. A.; Lehner, A. N.; Azami, M.; Barough, S. E.; Samadikuchaksaraei, A.; Antunes, A. P. M. The Effects of Crosslinkers on Physical, Mechanical, and Cytotoxic Properties of Gelatin Sponge Prepared Via In-Situ Gas Foaming Method as a Tissue Engineering Scaffold. Mat.Sci.Eng C 2016, 63, 1-9.

(20) Niknejad, H.; Mahmoudzadeh, R. Comparison of Different Crosslinking Methods for Preparation of Docetaxel-loaded Albumin Nanoparticles. Iran. J. Pharma.Res. 2015, 14, 385-394.

(21) Ghobril, C.; Grinstaff, M. W. The Chemistry and Engineering of Polymeric Hydrogel Adhesives for Wound Closure: A Tutorial. Chem.Soc.Rev. 2015, 44, 1820-1835.

(22) Cao, L.; Cao, B.; Lu, C.; Wang, G.; Yu, L.; Ding, J. An Injectable Hydrogel Formed By In Situ Crosslinking of Glycol Chitosan and Multi-Benzaldehyde Functionalized PEG Analogues for Cartilage Tissue Engineering. J. Mater. Chem. B 2015, 3, 1268 1280. 
(23) Fan, M.; Ma, Y.; Mao, J.; Zhang, Z.; Tan, H. Cytocompatible in Situ Forming Chitosan/Hyaluronan Hydrogels via a Metal-free Click Chemistry for Soft Tissue Engineering. Acta Biomater. 2015, 60-68.

(24) Deng, Y.; Ren, J.; Chen, G.; Li, G.; Wu, X.; Wang, G.; Gu, G.; Li, J. Injectable In Situ Forming Poly(L-glutamic acid) Hydrogels for Cartilage Tissue Engineering. Sci. Rep. 2017, 7, 2699-2711.

(25) Yan, S.; Zhang, X.; Zhang, K.; Di, H.; Feng, L.; Li, G.; Fang, J.; Cui, L.; Chen, X.; J Yin, J. Injectable In Situ forming Poly(L-Glutamic Acid) Hydrogels for Cartilage Tissue Engineering. J. Mater. Chem. B 2016, 4, 947-961.

(26) Li, Y.; Rodrigues, J.; Tomás, H. Injectable and Biodegradable Hydrogels: Gelation, Biodegradation and Biomedical Applications. Chem. Soc. Rev. 2012, 41, 2193-2221.

(27) Wang, X.; Tang, R.; Zhang, Y.; Yu, Z.; Qi, C. Preparation of a Novel Chitosan Based Biopolymer Dye and Application in Wood Dyeing. Polvmers 2016, 8, 338-350.

(28) Zheng, X.; Zhang, H.; She, Y.; Pu, J. Composite Films of N,O-Carboxymethyl Chitosan and Bamboo Fiber. L.Anol.Polvm. Sci. 2014, 131, 39851-39856.

(29) Wu, X.; He C.; Wu, Y.; Chen, X. Synergistic Therapeutic Effects of Schiff's Base Cross-Linked Injectable Hydrogels for Local Co-delivery of Metformin and 5Fluorouracil in a Mouse Colon Carcinoma Model. Biomaterials 2016, 75, 148-162.

(30) Jintapattanakit, A.; Mao, S.; Kissel T.; Junyaprasert, V. B. Physicochemical Properties and Biocompatibility of N-Trimethyl Chitosan: Effect of Quaternization and Dimethylation. Eur. J. Pharm. Biopharm. 2008, 70, 563-571.

(31) Kong, X. Simultaneous Determination of Degree of Deacetylation, Degree of Substitution and Distribution Fraction of - COONa in Carboxymethyl Chitosan by Potentiometric Titration. Carbohvdr Polvm. 2012, 88, 336-341.

(32) Zhou, X.; Zhang, X.; Zhou, J.; Li, L. An Investigation of Chitosan and Its Derivatives on Red Blood Cell Agglutination. RSC Adv. 2017, 7, 12247-12254.

(33) Mahou, R.; Wandrey, C. Versatile Route to Synthesize Heterobifunctional Poly(ethylene glycol) of Variable Functionality for Subsequent Pegylation. Polvm. 2012, 4, 561-589.

(34) Khazaei, A.; Abbasi, F.; Moosavi-Zare, A. M. Efficient Preparation of Some New 1Carbamatoalkyl-2-naphthols using N-halo Reagents in Neutral Media. RSC Adv. 2014, 4, 1388-1392. 
(35) ElShaer, A.; Hanson, P.; Worthington, T.; Lambert, P.; Mohammed, A. R. Preparation and Characterization of Amino Acids-Based Trimethoprim Salts. Pharmaceutics 2012, 4, 179-196.

(36) Yan, S.; Sun, Y.; Chen, A.; Liu, L.; Zhang, K.; Li, G. Duan, Y.; Yin, J. Templated Fabrication of pH-Responsive Poly(L-Glutamic Acid) Based Nanogels via SurfaceGrafting and Macromolecular Crosslinking. $\underline{R S C A d v}$. 2017, 7, 14888-14901.

(37) El-Ghaffar, M. A. A.; Hashem, M. S. Chitosan and Its Amino Acids Condensation Adducts as Reactive Natural Polymer Supports for Cellulase Immobilization. Carbohvdr. Polvm. 2010, 81, 507-516.

(38) Wang, Y.; Barry, A. P.; Habtemariam, A.; Canelon, A. R.; Sadler, P. J.; Barry, N. P. E. Nanoparticles of Chitosan Conjugated to Organoruthenium Complexes. Inorg. Chem. Front., 2016, 3, 1058-1064.

(39) Keong, L. C.; Halim, A. S. In Vitro Models in Biocompatibility Assessment for Biomedical-Grade Chitosan Derivatives in Wound Management. Int. J.Mol. Sci. 2009, 10, 1300-1313.

(40) Nie, J.; Wang, Z.; Zhang, K.; Hu, Q. Biomimetic Multi-Layered Hollow ChitosanTripolyphosphate Rod with Excellent Mechanical Performance. RSC Adv., 2015, 5, 37346-37352.

(41) Pizzoferrato, A.; Ciapetti, G.; Stea, S.; Cenni, E.; Arciola, R C.; Granchi, D.; Savarino, L. Cell Culture Methods for Testing Biocompatibility. Clin. Mater. 1994, $15,173-190$.

(42) Tan, H.; Chu, C. R.; Payne, K. A.; Marra, K. G. Injectable In Situ Forming Biodegradable Chitosan-Hyaluronic Acid Based Hydrogels for Cartilage Tissue Engineering. Biomaterials 2009, 30, 2499-2506.

(43) K. Sakurai, K.; Maegawa, T.; Takahashi, T. Glass Transition Temperature of Chitosan and Miscibility of Chitosan/Poly(N-vinyl pyrrolidone) Blends. Polvm. 2000, 41, 7051-7056.

(44) Santos, J. C. C.; Mansur, A. A. P.; Mansur, H. S. One-Step Biofunctionalization of Quantum Dots with Chitosan and N-palmitoyl Chitosan for Potential Biomedical Applications. Molecules 2013, 18, 6550-6572.

(45) Caddeo, C.; Nacher, A.; Díez-Sales, O.; Sanjuán, M. M.; Fadda, A. M.; Manconi, M. Chitosan-xanthan Gum Microparticle-Based Oral Tablet for Colon-Targeted and Sustained Delivery of Quercetin. L.Microencansul. 2014, 31, 694-699. 
(46) Suvannasara, P.; Siralertmukul, K.; Muangsin, N. Electrosprayed 4Carboxybenzenesulfonamide-Chitosan Microspheres for Acetazolamide Delivery. $\underline{\text { Int }}$. J. Biolog. Macromol. 2014, 64, 240-246.

(47) Kumar, S.; Koh, J. Physiochemical, Optical and Biological Activity of ChitosanChromone Derivative for Biomedical Applications. Int.J.Mol.Sci. 2012, 13, 61026116.

(48) Wang, Y.; Pitto-Barry, A.; Habtemariam, A.; Romero-Canelon, I.; Sadler, P. J.; Barry, N. P. E. Nanoparticles of Chitosan Conjugated to Organo-ruthenium Complexes. Inorg. Chem. Front. 2016, 3, 1058-1064.

(49) Kumar, R.; Isloor, A. M.; Ismail, A. F.; Matsuura, T. Synthesis and Characterization of Novel Water Soluble Derivative of Chitosanas an Additive for Polysulfone Ultrafiltration Membrane. L.Membr.Sci. 2013, 440, 140-147.

(50) Yan, S.; Wang, T.; Li, X.; Jian, Y.; Zhang, K.; Li, G.; Yin, J. Fabrication of Injectable Hydrogels Based on Poly(L-Glutamic Acid) and Chitosan. RSC Adv., 2017, 7, 17005-17019.

(51) Ahmad, M. B.; Tay, M. Y.; Shameli, K.; Hussein, M. Z.; Lim, J. I. Green Synthesis and Characterization of Silver/Chitosan/Polyethylene Glycol Nanocomposites without any Reducing. Int. J. Mol. Sci. 2011, 12, 4872-4884.

(52) Yin, G.; Chenac, G.; Zhou,Z.; Li, Q. Modification of PEG-b-PCL Block Copolymer with High Melting Temperature by the Enhancement of POSS Crystal and Ordered Phase Structure.. RSC Adv. 2015, 5, 33356-33363.

(53) Koivisto, J. T.; Joki, T.; Parraga, J. E.; Pääkkönen, R.; Ylä-Outinen, L.; Salonen, L.; Jönkkäri, I.; Peltola, M.; Ihalainen, T. O.; Narkilahti, S. Bioamine-Crosslinked Gellan Gum Hydrogel for Neural Tissue Engineering. Biomed. Mater. 2017;12, 025014.

(54) Tan, H.; Chu, C. R.; Payne, K. A.; Marra, K. G. Injectable In Situ Forming Biodegradable Chitosan-Hyaluronic Acid Based Hydrogels for Cartilage Tissue Engineering. Biomaterials 2009, 30, 2499-2506.

(55) Park, H.; Choi, B.; Hu, J.; Lee, M. Injectable Chitosan Hyaluronic Acid Hydrogels for Cartilage Tissue Engineering. Acta Biomater. 2013, 9, 4779-4786.

(56) Levental, I.; Georges, P. C.; Janmey, P. A. Soft Biological Materials and Their Impact on Cell Function. Soft Matter, 2007, 3, 299-306.

(57) Taylor, D. L.; Panhuis, M. H. Self-Healing Hydrogels. Adv. Mater. 2016, 28, 9060 9093. 
(58) Wu, Q. X.; Lin, D. Q.; Yao, S. J. Design of Chitosan and Its Water Soluble Derivatives-Based Drug Carriers with Polyelectrolyte Complexes. Mar. Drugs 2014, $12,6236-6253$.

(59) Guzman, J.; Saucedo, I.; Navarro, R.; Revilla, J.; Guibal, E. Vanadium Interactions with Chitosan: Influence of Polymer Protonation and Metal Speciation. Langmuir 2002, 18, 1567-1573.

(60) Pizzoferrato, A.; Ciapetti, G.; Stea, S.; Cenni, E.; Arciola, R C.; Granchi, D.; Savarino, L. Cell Culture Methods for Testing Biocompatibility. Clin. Mater. 1994, $15,173-190$.

(61) Nguyen, D. H.; Tran, N. Q.; Nguyen, C. K. Tetronic-grafted Chitosan Hydrogel as an Injectable and Biocompatible Scaffold for Biomedical Applications. J. Biomater. Sci. Polvm.Ed. 2013, 24, 1636-1648. 


\section{A Graphic for the Table of Contents}

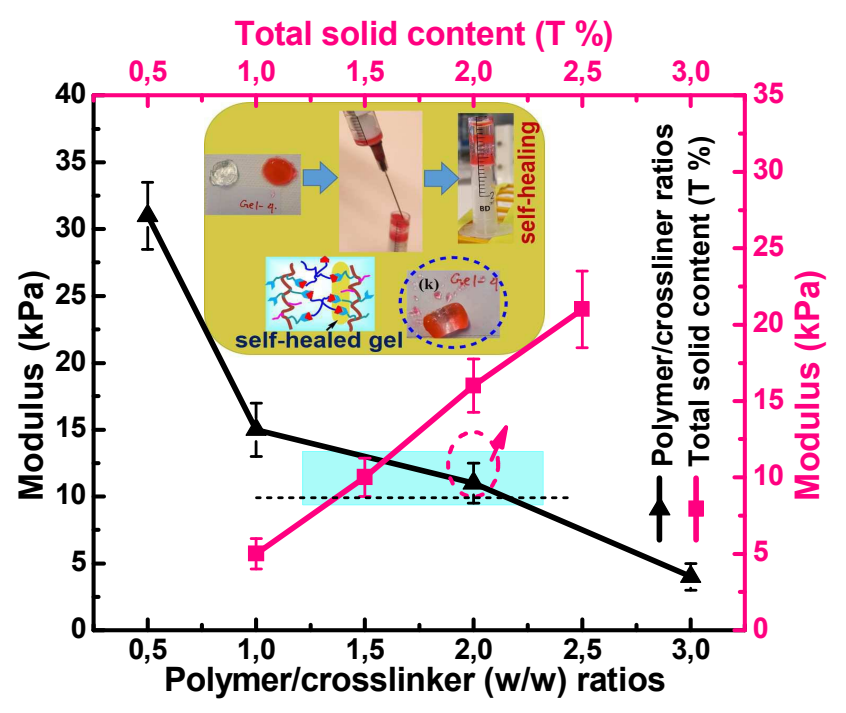

